\begin{tabular}{|c|c|}
\hline \multicolumn{2}{|c|}{ Statistica Sinica Preprint No: SS-2019-0410 } \\
\hline Title & $\begin{array}{l}\text { Penalized Jackknife Empirical Likelihood in High } \\
\text { Dimensions }\end{array}$ \\
\hline Manuscript ID & SS-2019-0410 \\
\hline URL & http://www.stat.sinica.edu.tw/statistica/ \\
\hline DOI & $10.5705 /$ ss.202019.0410 \\
\hline Complete List of Authors & $\begin{array}{l}\text { Zhouping Li, } \\
\text { Jinfeng Xu, } \\
\text { Na Zhao and } \\
\text { Wang Zhou }\end{array}$ \\
\hline Corresponding Author & Zhouping Li \\
\hline E-mail & zplistat@gmail.com \\
\hline Notice: Accepted version subje & ct to English editing. \\
\hline
\end{tabular}


Statistica Sinica

\title{
Penalized Jackknife Empirical Likelihood in High Dimensions
}

\author{
Zhouping $\mathrm{Li}^{1}$, Jinfeng $\mathrm{Xu}^{2}$, Na Zhao ${ }^{2}$ and Wang Zhou \\ ${ }^{1}$ Lanzhou University, ${ }^{2}$ The University of Hong Kong \\ and ${ }^{3}$ National University of Singapore
}

\begin{abstract}
Jackknife empirical likelihood (JEL) proposed by Jing et al. (2009) is an attractive approach for statistical inferences with nonlinear statistics such as $U$-statistics. However, most contemporary problems involve high dimensional model selection and its feasibility in theory and practice remains largely unexplored in the situations where the number of parameters diverges to infinity. In this paper, we propose a penalized JEL method which preserves the main advantages of JEL and leads to reliable variable selection based on the estimating equations with $U$-statistic structure in the high-dimensional setting. Under certain regularity conditions, we establish the asymptotic theory and oracle property for the JEL and its penalized version when the number of estimating equations and parameters increases along with the sample size. Simulation studies and real data analysis were carried out to examine the performance of the proposed methods and illustrate its practical utilities.

Key words and phrases: Estimating equations, high-dimensional data analysis, jackknife empirical likelihood, penalized likelihood, $U$-statistics, variable selection.
\end{abstract}




\section{Introduction}

Statistical inference based on estimating equations with $U$-statistics structure $(U$ type estimating equations) is common in nonparametric and semiparametric situations such as quantile regression and rank regression (Jin et al., 2003). Suppose that observations $X_{1}, \ldots, X_{n}$ are independent and identically distributed random vectors and the unknown parameters $\theta=\left(\theta_{1}, \ldots, \theta_{p}\right)^{T}$ can be estimated by solving the following $r(r \geq p)$ estimating equations

$$
U_{n}(\theta)=\left(\begin{array}{l}
n \\
k
\end{array}\right)^{-1} \sum_{1 \leq i_{1}<\ldots<i_{k} \leq n} h\left(X_{i_{1}}, \ldots, X_{i_{k}} ; \theta\right)=0
$$

where $h(\cdot)=\left(h_{1}(\cdot), \ldots, h_{r}(\cdot)\right)^{T}$ are symmetric in $X=\left(X_{1}, \ldots, X_{k}\right)^{T}$ 's, and satisfy $E h\left(X_{1}, \ldots, X_{k} ; \theta_{0}\right)=0$ with $\theta_{0}$ which denotes the true value of $\theta$. Here $\Sigma$ denotes the summation over subsets of $k$ integers $\left\{i_{1}, \ldots, i_{k}\right\}$ from $\{1, \ldots, n\}$. For many estimation procedures in the literature, the estimator $\hat{\theta}$ is formally defined as the solution of the above $U$-type estimating equations $(\mathbb{\square} . \mathbb{})$, which is known as a $U$-type estimating problem. See Jin et al. (2001), Song and Ma (2010), Li et al. (2016), among others. In this paper, we are interested in this problem under the high-dimensional setting, that is, $p$ and $r$ diverge with the sample size $n$. Hence we use $p_{n}$ and $r_{n}$ throughout the paper to emphasize the dependence of $p$ and $r$ on $n$.

High-dimensional data have now become ubiquitous in many applications such as 
microarray data analysis, neuroimaging, and portfolio selection, where the number of parameters or variables, $p_{n}$, is very large, usually in thousands or beyond. Penalized methods are effective in analyzing such data, and various penalty functions have been proposed including the lasso (Tibshirani, 1996), the SCAD (Fan and Li, 2001), the adaptive lasso (Zou, 2006), least-squares approximation (Wang and Leng, 2007), and so on. Although these methodologies significantly improved the inference and variable selection procedures in the high-dimensional settings such as linear regression and generalized linear models, to our best knowledge, its feasibility in jackknife

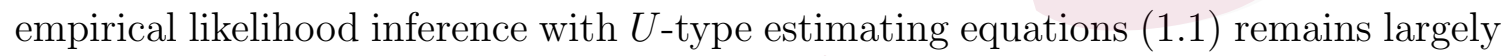
unexplored.

The empirical likelihood (EL) method introduced by Owen $(1988,1990)$, has been extensively studied and widely used to construct confidence regions and to test hypotheses in the literature. One nice feature of this method is that the confidence intervals and $p$-values of a test can be easily obtained without estimating the covariance matrix. More details can be found in Owen (2001), Chen and Van Keilegom (2009). Notice that the standard EL method works well in dealing with linear constraints, and an effective way of formulating the EL ratio statistic is via estimating equations as in Qin and Lawless (1994), while for nonlinear constraints, the EL method is extremely complicated in computation. To overcome the computational difficulty, Jing et al. (2009) proposed jackknife empirical likelihood (JEL) with 
particular attention on nonlinear statistics involving $U$-statistics. Subsequently, Li et al. (2011), and Peng (2012) extended JEL to general estimating equations. Recently, high-dimensional data analysis incorporating with EL method attracted more attentions, and was investigated by Hjort et al. (2009), Chen et al. (2009), Tang and Leng (2010), Lahiri and Mukhopadhyay (2012), Leng and Tang (2012), Peng et al. (2014), Chang et al. (2015), Chen et al. (2015), Li et al. (2017), Chang et al. (2018), Wang et al. (2019), Tang et al. (2020), Chang et al. (2021) and references therein. Motivated by these developments, when dimension grows with the sample size $n$, simultaneous estimation of parameters and variable selection using $U$-type estimating equations $(\mathbb{\square} . \mathbb{\text { l }})$ is of great interest and challenging, both in theory and computation. Our theoretical results can be summarized as two-fold.

(1). We prove that JEL method is efficient when dealing with high-dimensional $U$ type estimating equations, and provide the corresponding algorithms in this scenario, which extends the scope of the JEL methods for $U$-type estimating equations from fixed dimensions ( $\mathrm{Li}$ et al., 2016) to the case of diverging dimensions.

(2). We propose a novel penalized JEL (PJEL) approach based on the $U$-type estimating equations. By choosing the proper penalty function, the approach preserves the main advantages of the JEL and the penalized method and the resulting estimator possesses good properties such as the oracle property, cor- 
rectly select the true sparse model with probability tending to one and with optimal efficiency. Also, Wilks' theorem continues to hold for constructing confidence regions and testing hypotheses.

The rest of the paper is organized as follows. Section 2 presents the JEL method in high-dimensional settings. In Section 3, we describe the PJEL methodology and obtain its asymptotic theories. In Sections 4 and 5, we further report simulation results as well as a real data application to assess its finite-sample performance and illustrate its practical utility. All proofs are relegated to the Appendix.

\section{Jackknife empirical likelihood with a diverging number of parameters}

For the $U$-type estimating problem, the estimator $\hat{\theta}$ of $\theta_{0}$ is the solution to $U_{n}^{T}(\theta)=$ $\left(U_{n, 1}(\theta), \ldots, U_{n, r_{n}}(\theta)\right)^{T}=0$, where

$$
U_{n, l}(\theta)=\left(\begin{array}{l}
n \\
k
\end{array}\right)^{-1} \sum_{1 \leq i_{1}<\ldots<i_{k} \leq n} h_{l}\left(X_{i_{1}}, \ldots, X_{i_{k}} ; \theta\right), \quad l=1, \ldots, r_{n}
$$

As the straightforward application of the standard EL method involves many nonlinear constraints, which causes heavy computational burden, and thus is not favorable. To overcome this difficulty, Jing et al. (2009) proposed to first obtain $n$ jackknife pseudo values and then apply the standard EL to the nonlinear constraints in $U$-statistics. They demonstrated that this procedure is particular efficient in this 
situation. However, their discussion is restricted to the estimation of the mean of one-sample and two-sample statistics, and the dimension is fixed, in this paper we propose a general JEL procedure for inferences with this $U$-type estimation problem which avoids the nonlinear constraints and permits the high-dimensional estimating equations. The method is constructed based on the fact that $U_{n}(\theta)$ is an unbiased and consistent estimator of $E h\left(X_{1}, \ldots, X_{k} ; \theta\right)$ and has mean zero at the true parameter value $\theta_{0}$. The details are as follows.

Define $T_{n}=U_{n}(\theta)$ and $T_{n-1}^{(-i)}=T\left(X_{1}, \ldots, X_{i-1}, X_{i+1}, \ldots, X_{n} ; \theta\right)$, the statistic computed on the original data set with the $i$ th observation removed. The jackknife pseudo-values

$$
\widehat{V}_{i}(\theta)=n T_{n}-(n-1) T_{n-1}^{(-i)}
$$

can be shown to be asymptotically independent under mild conditions. As they also unbiasedly and consistently estimate $E h\left(X_{1}, \ldots, X_{k} ; \theta\right)$, a standard empirical likelihood can then be constructed on $\widehat{V}_{i}(\theta), i=1, \ldots, n$, instead of the original observations $X_{1}, \ldots, X_{n}$ as follows. Specifically, the JEL function is defined as

$$
\mathcal{L}(\theta)=\max \left\{\prod_{i=1}^{n} p_{i}: \sum_{i=1}^{n} p_{i}=1, \sum_{i=1}^{n} p_{i} \widehat{V}_{i}(\theta)=0\right\}
$$


with the corresponding likelihood ratio

$$
\mathcal{R}(\theta)=\max \left\{\prod_{i=1}^{n}\left(n p_{i}\right): \sum_{i=1}^{n} p_{i}=1, \sum_{i=1}^{n} p_{i} \widehat{V}_{i}(\theta)=0\right\}
$$

Throughout the paper, $\|\cdot\|$ always refers to the $l_{2}$-norm $\|\cdot\|_{2}, q$ is any fixed integer. In the Appendix, we prove the following theorems.

Theorem 1. Let $\hat{\theta}_{E}$ be the minimizer of (2.4). Under Conditions C1-C5 in the Appendix, as $n \rightarrow \infty$ and with probability tending to 1 , we have

(i) $\hat{\theta}_{E} \stackrel{p}{\rightarrow} \theta_{0}$, (ii) $\left\|\hat{\theta}_{E}-\theta_{0}\right\|=O_{p}\left(a_{n}\right)$, where $a_{n}$ is given in the Appendix.

Theorem 2. Under Conditions C1-C5 in the Appendix, we have

$$
A_{n} \Omega_{22.1}^{1 / 2} \sqrt{n}\left(\hat{\theta}_{E}-\theta_{0}\right) \stackrel{d}{\rightarrow} N(0, \Delta)
$$

where $A_{n} \in R^{q \times p_{n}}$ such that $A_{n} A_{n}^{T} \rightarrow G$ and $G$ is a $q \times q$ nonnegative matrix, $\Omega_{22.1}$ is given in the Appendix.

\section{Penalized jackknife empirical likelihood}

In this section, we establish the asymptotic theory for the PJEL estimator. Suppose that $\theta$ can be partitioned as $\theta=\left(\theta_{1}^{T}, \theta_{2}^{T}\right)^{T}$, where $\theta_{1} \in R^{d}$ and $\theta_{2} \in R^{p_{n}-d}$ corre- 
sponding to the nonzero and zero components, respectively. Then the true parameter value $\theta_{0}=\left(\theta_{10}^{T}, \mathbf{0}\right)^{T}$. We estimate the unknown parameter vector $\theta_{0}$ by minimizing

$$
l_{p}(\theta)=\sum_{i=1}^{n} \log \left\{1+\lambda^{T} \widehat{V}_{i}(\theta)\right\}+n \sum_{j=1}^{p_{n}} p_{\tau}\left(\left|\theta_{j}\right|\right)
$$

where $p_{\tau}\left(\left|\theta_{j}\right|\right)$ is some penalty function, $\tau$ is a tuning parameter that controls the trade-off between bias and model complexity (see Fan and Li, 2001). The PJEL has the following oracle property.

Theorem 3. Let $\hat{\theta}=\left(\hat{\theta}_{1}^{T}, \hat{\theta}_{2}^{T}\right)^{T}$ be the minimizer of (B.5). Under Conditions C1-Cry in the Appendix, as $n \rightarrow \infty$, we have

(i). $P\left(\hat{\theta}_{2}=0\right) \rightarrow 1$;

(ii). $\quad n^{1 / 2} B_{n} \Delta_{11.2}^{-1 / 2}\left(\hat{\theta}_{1}-\theta_{10}\right) \stackrel{d}{\rightarrow} N\left(0, G^{*}\right)$, where $B_{n}$ is a $q \times d$ matrix such that $B_{n} B_{n}^{T} \rightarrow G^{*}, G^{*}$ is a $q \times q$ nonnegative symmetric matrix, $\Delta_{11.2}$ is given in the Appendix.

As the number of parameters may diverge to infinity, we next study the problem of testing the following linear hypothesis:

$$
H_{0}: L_{n} \theta_{10}=0, \quad \text { v.s. } H_{1}: L_{n} \theta_{10} \neq 0
$$

where $L_{n}$ is a $q \times d$ matrix such that $L_{n} L_{n}^{T}=I_{q}$ for fixed $q$. Noting that this type of 
hypothesis includes simultaneously testing whether a few variables are statistically significant. In the penalized likelihood context, Fan and Peng (2004) investigated this type of hypothesis testing in the parametric likelihood framework, and Leng and Tang (2012) lately considered this problem in a standard empirical likelihood settings for general estimating equations. Our results further generalized those existing results. Specifically, a PJEL ratio test statistic is defined as

$$
\tilde{l}\left(L_{n}\right)=-2\left\{l_{p}(\hat{\theta})-\min _{\theta_{1}, L_{n} \theta_{1}=0} l_{p}(\theta)\right\}
$$

The following theorem derives the asymptotic null distribution of the above test statistic.

Theorem 4. Under the null hypothesis and Conditions C1-C7 in the Appendix, we have

$$
\tilde{l}\left(L_{n}\right) \stackrel{d}{\rightarrow} \chi_{q}^{2}, \quad \text { as } n \rightarrow \infty
$$

From Theorem 4, a $(1-\alpha)$-level confidence region for $L_{n} \theta_{1}$ can be constructed as

$$
C_{\alpha}=\left\{v:-2\left\{l_{p}(\hat{\theta})-\min _{\theta_{1}, L_{n} \theta_{1}=v} l_{p}(\theta)\right\} \leq \chi_{q, 1-\alpha}^{2}\right\}
$$

where $\chi_{q, 1-\alpha}^{2}$ is the $(1-\alpha)$ th quantile of the $\chi_{q}^{2}$ distribution.

Finally, the algorithms for implementing the proposed PJEL method and con- 
structing the related confidence region are summarized as follows.

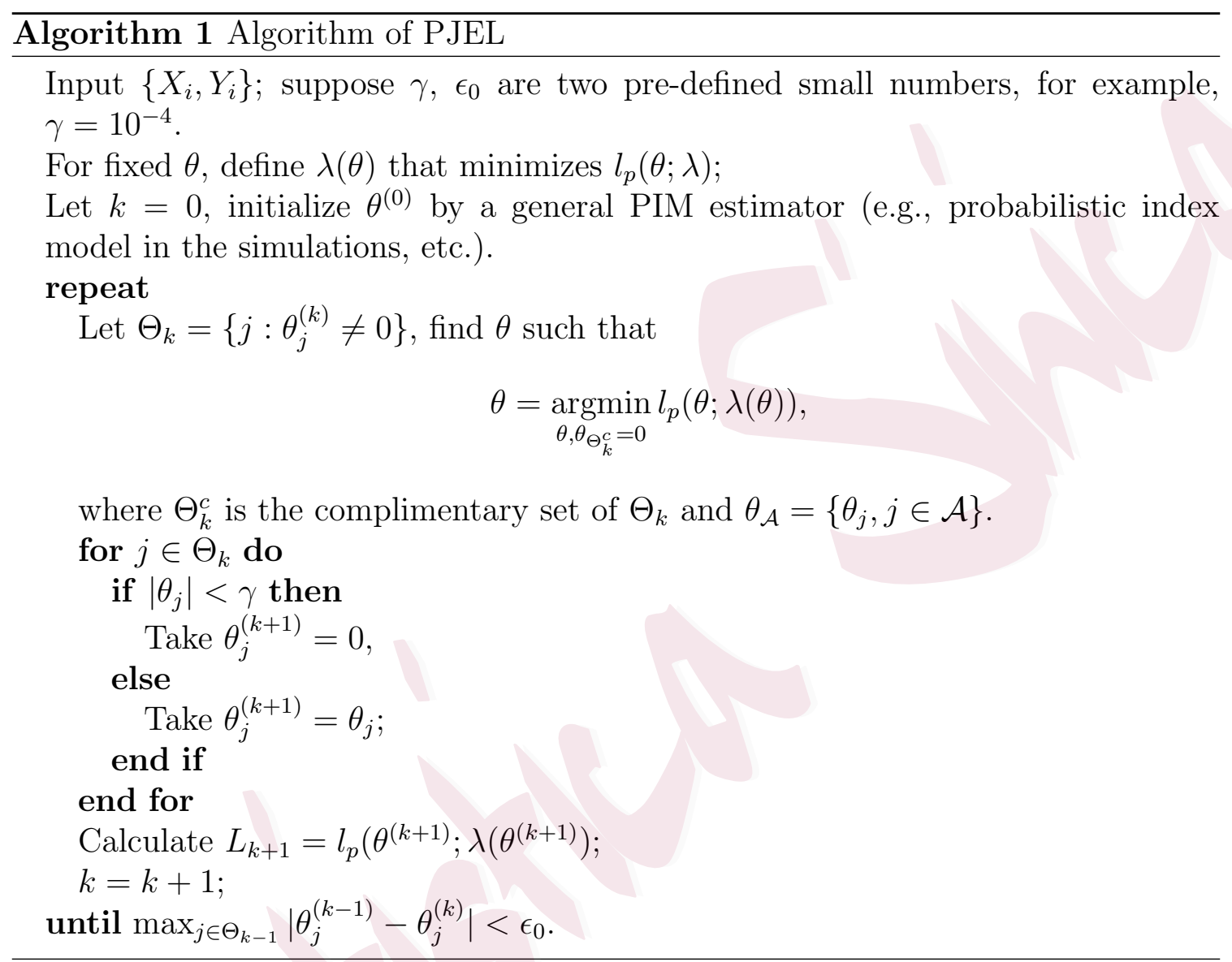




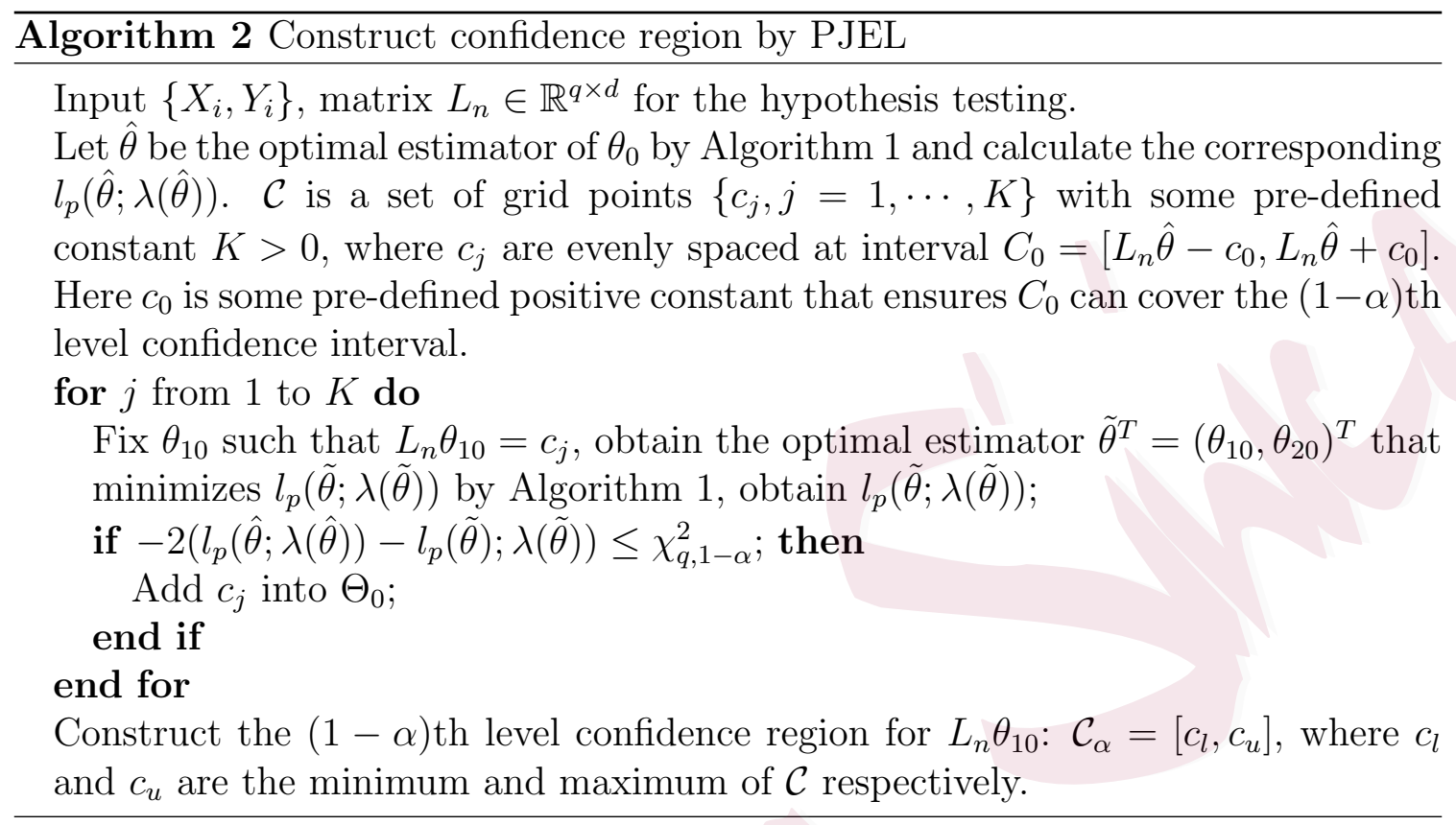

\section{Simulation studies}

In this section, we use the probabilistic index model (PIM) (Thas et al, 2012) as an example to illustrate the proposed method for $U$-structured problems. The effect of the covariates $X$ on the response $Y$ is evaluated through the probabilistic index, which is defined as the probability $\mathrm{P}\left(Y_{i} \preccurlyeq Y_{j}\right):=\mathrm{P}\left(Y_{i}<Y_{j}\right)+0.5 \mathrm{P}\left(Y_{i}=Y_{j}\right)$, where $Y_{i}$ and $Y_{j}$ are independent response variables with identical distribution function $F$. Data consist of i.i.d. observations $\left(Y_{i}, X_{i}\right), i=1, \ldots, n$. A PIM is defined as:

$$
\mathrm{P}\left(Y_{i} \preccurlyeq Y_{j} \mid X_{i}, X_{j}\right)=m\left(X_{i}, X_{j} ; \beta\right)=g^{-1}\left(Z_{i j}^{T} \beta\right)
$$


where $g(\cdot)$ is a link function and $Z_{i j}$ depends on $X_{i}$ and $X_{j}$. Following Thas et al (2012), let $Z_{i, j}=X_{j}-X_{i}$ and for the probit and logit link function, the model is termed as the linear PIM and Cox PIM, respectively. De Neve and Thas (2015) proposed the $U$-type estimating equations:

$$
\sum_{(i, j)} U_{i j}(\beta)=\mathbf{0}, \quad U_{i j}(\beta):=Z_{i j}\left[\mathrm{I}\left(Y_{i} \preccurlyeq Y_{j}\right)-g^{-1}\left(Z_{i j}^{T} \beta\right)\right]
$$

As in Thas et al (2012), we consider two scenarios as follows.

(a) Normal linear model: $Y_{i} \mid X_{i}$ are i. i. d. $N\left(\alpha_{1} X_{1 i}+\alpha_{2} X_{2 i}, 1\right)$. In this setting, the corresponding PIM is given by $\Phi^{-1}\left\{P\left(Y_{i} \preccurlyeq Y_{j} \mid X_{i} X_{j}\right)\right\}=\beta_{1}\left(X_{1 j}-X_{1 i}\right)+\beta_{2}\left(X_{2 j}-X_{2 i}\right)$, where $\beta_{i}=\alpha_{i} / \sqrt{2}$ and $\Phi$ is the distribution function of a standard normal distribution.

(b) Exponential model: $Y_{i} \mid X_{i}$ are i.i.d. Exponential $\left\{\exp \left(\alpha_{1} X_{1 i}+\alpha_{2} X_{2 i}\right)\right\}$. In this setting, the corresponding PIM is given by $\operatorname{logit}\left\{P\left(Y_{i} \preccurlyeq Y_{j} \mid X_{i} X_{j}\right)\right\}=\beta_{1}\left(X_{1 j}-X_{1 i}\right)+$ $\beta_{2}\left(X_{2 j}-X_{2 i}\right)$, where $\beta_{i}=-\alpha_{i}$.

In the above settings, the covariate $X_{1}$ is a Bernoulli random variable with success probability 0.5 and, $X_{2}$ follows $U[0,10]$, and $\alpha_{1}=1, \alpha_{2}=1$. For various combinations of $p$ and $n$, simulations were conducted with 1000 repetitions.

Tuning parameters $\tau$ is taken from a fine grid and chosen by the BIC-type crite- 
rion (Wang et al., 2009)

$$
B I C(\tau)=2 \ell_{p}\left(\beta_{\tau}\right)+C_{n} \log n d f_{\tau}
$$

where $\beta_{\tau}$ is the PJEL with tuning parameter $\tau$ and $d f_{\tau}$ is the number of nonzero coefficient in $\beta_{\tau}$. For fixed $p, C_{n}=1$ and for diverging $p, C_{n}=\max \{\log \log p, 1\}$. In the numerical study, we find that the selected tuning parameter for BIC decreases as sample size increases, while the remaining parameters like covariates dimension and value of $\beta$ keep the same. Also, the ratio of $\lambda$ to the square root of $p / n$ increases along with the sample size (not shown in the tables). The results are consistent with the regularized condition in our assumptions, which validates the BIC-type criterion here.

We also compare the proposed method with penalized empirical likelihood (PEL) introduced by Tang and Leng (2010), and penalized maximum smoother rank correlation (PMSRC) introduced by Lin and Peng (2013), using the same datasets and tuning criteria. Since the method in Tang and Leng (2010) only considered the linear constraints case, we first use the sequential linearization method in Wood et al. (1996) to linearize the nonlinear constraints, and then applied the PEL to it. The latter method achieved the estimation through a penalized smoothing objective function for maximum rank correlation, which is a typical $U$-statistics. The simulation 


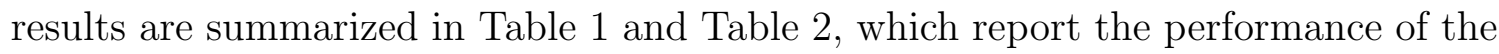
proposed method in terms of the median of $L_{2}$ distance (MD) of the estimation, the average correctly selected zero coefficients (C) and the average incorrectly estimated zero coefficients (IC) for variable selection. It can be seen that in both scenarios, the PJEL method performs well in both the estimation and variable selection. Moreover, it identified most of the true zero-coefficients to be zero, while for PEL, the performance is not good enough for the variable selection. For PMSRC, although the estimation is quite good in linear PIM model, for Cox model, the performance is not as good as PJEL. Moreover, the variable selection capability is not comparable to the PJEL. Also, in order to illustrate the computational improvement of introducing jackknife method to EL model, we report the average computational time of PJEL and PEL in the above setting for per simulation in Table 3 . From the table, we can find that, in both linear and Cox PIM models, the time PEL spent is much larger than that of PJEL, and when the sample size or covariates dimension grows, the ratio of the time expenditure between these two methods is growing larger. This indicates that when we are dealing with large sample size dataset via $U$-statistic structure estimating equations, with high dimension covariates, PEL is not applicable due to the computational burden. This reveals the advantage of the PJEL method compared to the PEL method in terms of the computaional efficiency. 
Table 1: Simulation results for linear PIM model with PJEL, PEL and PMSRC methods.

\begin{tabular}{|c|c|c|c|c|c|c|c|c|c|c|}
\hline \multirow[b]{2}{*}{$n$} & \multirow[b]{2}{*}{$p$} & \multicolumn{3}{|c|}{ PJEL } & \multicolumn{3}{|c|}{ PEL } & \multicolumn{3}{|c|}{ PMSRC } \\
\hline & & MD & $\mathrm{C}$ & $\mathrm{IC}$ & $\mathrm{MD}$ & $\mathrm{C}$ & $\mathrm{IC}$ & MD & $\mathrm{C}$ & $\mathrm{IC}$ \\
\hline 200 & 5 & 0.0548 & 2.936 & 0.000 & 0.0527 & 2.346 & 0.000 & 0.0354 & 2.442 & 0.000 \\
\hline 300 & 10 & 0.0418 & 7.934 & 0.000 & 0.0392 & 5.878 & 0.000 & 0.0295 & 6.763 & 0.000 \\
\hline 400 & 15 & 0.0378 & 12.918 & 0.000 & 0.0366 & 9.395 & 0.000 & 0.0207 & 11.275 & 0.000 \\
\hline
\end{tabular}

Table 2: Simulation results for Cox PIM model with PJEL, PEL and PMSRC methods.

\begin{tabular}{|c|c|c|c|c|c|c|c|c|c|c|}
\hline \multirow[b]{2}{*}{$n$} & \multirow[b]{2}{*}{$p$} & \multicolumn{3}{|c|}{ PJEL } & \multicolumn{3}{|c|}{ PEL } & \multicolumn{3}{|c|}{ PMSRC } \\
\hline & & MD & $\mathrm{C}$ & IC & MD & $\mathrm{C}$ & $\mathrm{IC}$ & MD & $\mathrm{C}$ & $\mathrm{IC}$ \\
\hline 200 & 5 & 0.0647 & 2.930 & 0.053 & 0.0850 & 2.278 & 0.000 & 0.1252 & 2.112 & 0.000 \\
\hline 300 & 10 & 0.0551 & 7.905 & 0.000 & 0.0548 & 6.598 & 0.000 & 0.1058 & 6.245 & 0.000 \\
\hline 400 & 15 & 0.0446 & 12.904 & 0.000 & 0.0460 & 9.732 & 0.000 & 0.0919 & 7.246 & 0.000 \\
\hline
\end{tabular}

Table 3: The average consumption time (in seconds) for PJEL and PEL with deterministic sample size $n$ and covariates $p$.

\begin{tabular}{|ccrrrrr|}
\hline \multirow{2}{*}{$n$} & $p$ & \multicolumn{2}{c}{ Linear PIM } & & \multicolumn{2}{c|}{ Cox PIM } \\
\cline { 5 - 6 } & & PJEL & PEL & & PJEL & PEL \\
\hline 200 & 5 & 4.140 & 16.394 & & 5.236 & 34.895 \\
300 & 10 & 18.093 & 115.586 & & 40.399 & 344.050 \\
400 & 15 & 58.542 & 451.907 & & 191.416 & 1692.695 \\
\hline
\end{tabular}

Next, we illustrate the performance of PJEL in constructing confidence region. We set $L_{n}$ in (‥6) to be $(1,0, \cdots, 0)$, and take the null hypothesis testing $H_{0}: \beta_{10}=a$ with $a=\beta_{1}-0.2, \beta_{1}-0.1, \beta_{1}, \beta_{1}+0.1, \beta_{1}+0.2$ separately, where $\beta_{10}$ denotes the first component of $\beta$. Under the nominal level $\alpha_{0}=0.05$, we summarize the empirical size for deterministic value of $a$ in Table 田. From the table, we can see the size of 
our test is close to the nominal level, and increases when the null value differs from the true value $\beta_{1}$. The results validate the likelihood ratio test under PJEL, and indicates the feasibility to construct confidence region by taking hypothesis at finer grid points.

Table 4: The empirical percentages that a given value does not fall in the $95 \%$ confidence interval

\begin{tabular}{|cccccccc|}
\hline PIM model & $n$ & $p$ & $\beta_{10}-0.2$ & $\beta_{10}-0.1$ & $\beta_{10}$ & $\beta_{10}+0.1$ & $\beta_{10}+0.2$ \\
\hline \multirow{3}{*}{ Normal linear } & 200 & 5 & 24.40 & 11.20 & 4.60 & 10.00 & 26.10 \\
& 300 & 10 & 40.40 & 15.80 & 6.40 & 12.00 & 33.80 \\
& 400 & 15 & 54.70 & 22.50 & 4.70 & 12.20 & 40.70 \\
\hline \multirow{3}{*}{ Exponential } & 200 & 5 & 26.90 & 14.10 & 6.50 & 9.10 & 16.80 \\
& 300 & 10 & 31.70 & 15.60 & 6.30 & 10.10 & 21.40 \\
& 400 & 15 & 41.60 & 20.00 & 6.90 & 10.60 & 26.70 \\
\hline
\end{tabular}

\section{An application to the air pollution study}

Adverse effects of air pollution on human health have been explored in many scientific studies. In particular, it is of great interest to evaluate the effect of air pollution on human mortality. Here, we apply the proposed method to the air pollution study (McDonald et al., 1973) to identify the factors associated with air that affect the mortality the most. The dataset consists of 60 observations and 15 features. The response variable is "Mortality", representing the total age-adjusted mortality rate per 100,000. A detailed description of 15 variables in the air pollution study is given 
in Table 5 .

Table 5: A description of 15 variables in the air pollution study

\begin{tabular}{|ll|}
\hline PREC & Average annual precipitation in inches \\
JANT & Average January temperature in degrees F \\
JULT & Average July temperature in degrees F \\
OVR65 & of 1960 SMSA population aged 65 or older \\
POPN & Average household size \\
EDUC & Median school years completed by those over 22 \\
HOUS & of housing units which are sound \& with all facilities \\
DENS & Population per sq. mile in urbanized areas, 1960 \\
NONW & non-white population in urbanized areas, 1960 \\
WWDRK & employed in white collar occupations \\
POOR & of families with income less than $\$ 3000$ \\
HC & Relative hydrocarbon pollution potential \\
NOX & Same for nitric oxides \\
SO_2 & Same for sulphur dioxide \\
HUMID & Annual average relative humidity at $1 \mathrm{pm}$ \\
\hline
\end{tabular}

McDonald et al. (1973) proposed to use multiple linear regression to assess the covariate effects on the mortality rate. Here, apply the proposed method to fit a normal linear PIM, to identify the factors which are relevant to the mortality. The BIC-type criterion is used to choose the parameter $\lambda$. Then using the penalized jackknife empirical likelihood method, six covariates are identified to be relevant ones and reported in Table [6 along with the constructed $95 \%$ confidence intervals by the penalized JEL procedures for inference. The identified covariates are: average annual precipitation (PREC), average January temperature (JANT), median school years completed over 22 years (EDU), percent non-white population (NONW), hydrocarbon pollution potential(HC) and nitrous oxide pollution potential (NOX). The variables 
selected here are similar to the ones by McDonald et al. (1973). They used two different criteria to choose the factors. For the result of using ridge regression, out of 7 features they chose, we have 4 of them in common (annual precipitation, January temperature,education, and non-white percentage). In addition, we find that the hydrocarbon as well as nitric oxides pollution potential also have adverse effects on the human mortality rate.

Table 6: 95\% confidence intervals of the estimated non-zero coefficients for air pollution study.

\begin{tabular}{|ccc|}
\hline Covariate & Estimated coefficient & Confidence interval by PJEL \\
\hline PREC & 0.731 & {$[0.361,1.203]$} \\
JANT & -1.113 & {$[-1.472,-0.822]$} \\
EDUC & -0.390 & {$[-0.712,-0.161]$} \\
NONW & 1.743 & {$[1.36,2.23]$} \\
HC & -3.542 & {$[-5.612,-2.65]$} \\
NOX & 3.956 & {$[2.096,5.89]$} \\
\hline
\end{tabular}

\section{Supplementary Material}

The Supplementary Material includes detailed proofs of the main theorems.

\section{Acknowledgements}

The authors sincerely thank the Editor, an Associate Editor, and two anonymous reviewers for their very helpful and constructive comments that have led to significant improvements in the paper. Zhouping Li's research was supported by the National 
Natural Science Foundation of China (11571154), Jinfeng Xu and Na Zhao's research was supported by National Natural Science Foundation of China (72033002), the University of Hong Kong-Zhejiang Institute of Research and Innovation Seed Fund, and General Research Fund (17306619, 17308018 and 17308820) of Hong Kong, Wang Zhou's research was partially supported by a grant R-155-000-192-114 at the National University of Singapore.

\section{References}

Chang, J., Chen, S. and Chen, X. (2015). High dimensional generalized empirical likelihood for moment restrictions with dependent data. Journal of Econometrics 185, 283-304.

Chang, J., Chen, S., Tang, C. and Wu, T. (2021). High-dimensional empirical likelihood inference. Biometrika 108, 127-147.

Chang, J., Tang, C. and Wu, T. (2018). A new scope of penalized empirical likelihood with high-dimensional estimating equations. The Annals of Statistics 46, 3185-3216.

Chen, B., Pan, G., Yang, Q. and Zhou, W. (2015). Large dimensional empirical likelihood. Statisitica Sinica 25, 1659-1677.

Chen, S. X., Peng, L. and Qin, Y.L. (2009). Effects of data dimension on empirical likelihood. Biometrika 96, 711-722.

Chen, S. X. and Van Keilegom, I. (2009). A review on empirical likelihood methods for regression (with Discussion). Test 18, 415-447. 
Cui, X., Li, R., Yang, G. and Zhou, W. (2020). Empirical likelihood test for a large-dimensional mean vector. Biometrika 107, 591-607.

De Neve, J. and Thas, O. (2015). A regression framework for rank tests based on the probabilistic index model. Journal of the American Statistical Association 110, 1276-1283.

Fan, J. and Li, R. (2001). Variable selection via nonconcave penalized likelihood and its oracle properties. Journal of the American Statistical Association 96, 1348-1360.

Fan, J. and Peng, H. (2004). Nonconcave penalized likelihood with a diverging number of parameters. The Annals of Statistics 32, 928-961.

Hjort, N. L., McKeague, I. and Van Keilegom, I. (2009). Extending the scope of empirical likelihood. The Annals of Statistics 37, 1079-1111.

Hoeffding, W. (1948). A class of statistics with asymptotically normal distribution. The Annals of Mathematical Statistics 19, 293-325.

Huang, J., Horowitz, J. L. and Ma, S. (2008). Asymptotic properties of bridge estimators in sparse highdimensional regression models. The Annals of Statistics 36, 587-613.

Jin, Z., Ying, Z. and Wei, L. (2001). A simple resampling method by perturbing the minimand. Biometrika 88, 381-390.

Jin, Z., Lin, D.Y., Wei, L. J., and Ying, Z. (2003). Rank-based inference for the accelerated failure time models. Biometrika 90, 341-353.

Jing, B.-Y., Yuan, J. and Zhou, W. (2009). Jackknife empirical likelihood. Journal of the American Statis- 
tical Association 104, 1224-1232.

Lahiri, S. N. and Mukhopadhyay, S. (2012). A penalized empirical likelihood method in high dimensions. The Annals of Statistics 40, 2511-2540.

Lee, A.J. (1990). U-statistics, Theory and Practice. New York: Marcel Dekker, Inc.

Leng, C. and Tang, C. (2012). Penalized empirical likelihood and growing dimensional general estimating equations. Biometrika 99, 703-716.

Li, M., Peng, L. and Qi, Y. (2011). Reduce computation in profile empirical likelihood method. The Canadian Journal of Statistics 39, 370-384.

Li, Z., Liu, Y. and Liu, Z. (2017). Empirical likelihood and general relative error criterion with divergent dimension. Statistics 51, 1006-1022.

Li, Z., Xu, J. and Zhou, W. (2016). On nonsmooth estimating functions via jackknife empirical likelihood. Scandinavian Journal of Statistics 43, 49-69.

Lin, H. and Peng, H. (2013). Smoothed rank correlation of the linear transformation regression model. Computational Statistics and Data Analysis 57, 615-630.

McDonald, G. C. and Schwing, R. C. (1973). Instabilities of regression estimates relating air pollution to mortality. Technometrics 15, 463-481.

Newey, W. K. and Smith, R. J. (2004). Higher order properties of GMM and generalized empirical likelihood estimators. Econometrica 72, 219-255.

Owen, A.B. (1988). Empirical likelihood ratio confidence intervals for a single functional. Biometrika $\mathbf{7 5}$, 
237-249.

Owen, A.B. (1990). Empirical likelihood ratio confidence regions. The Annals of Statistics 18, 90-120.

Owen, A.B. (2001). Empirical Likelihood. Chapman and Hall, London.

Peng, L. (2012). Approximate jackknife empirical likelihood method for estimating equations. The Canadian Journal of Statistics 40, 110-123.

Peng, L., Qi, Y. and Wang, R. (2014). Empirical likelihood test for high dimensional linear models. Statistics \& Probability Letters 86, 85-90.

Qin, J. and Lawless, J.F. (1994). Empirical likelihood and general estimating equations. The Annals of Statistics 22, 300-325.

Song, X. and Ma, S. (2010). Penalized variable selection with U-estimates. Journal of Nonparametric Statistics 22, 499-515.

Tang, C. and Leng, C. (2010). Penalized high-dimensional empirical likelihood. Biometrika 97, 905-920.

Tang, N., Yan, X. and Zhao, X. (2020). Penalized generalized empirical likelihood with a diverging number of general estimating equations for censored data. The Annals of Statistics 48, 607-627.

Thas, O., Neve, J. D., Clement, L. and Ottoy, J. P. (2012). Probabilistic index models. Journal of the Royal Statistical Society: Series B (Statistical Methodology) 74, 623-671.

Tibshirani, R. J. (1996). Regression shrinkage and selection via the LASSO. Journal of the Royal Statistical Society: Series B (Statistical Methodology) 58, 267-288.

Wang, H. and Leng, C. (2007). Unified Lasso estimation via least squares approximation. Journal of the 


\section{REFERENCES}

American Statistical Association 102, 1039-1048.

Wang, H., Li, B. and Leng, C. (2009). Shrinkage tuning parameter selection with a diverging number of parameters. Journal of the Royal Statistical Society: Series B (Statistical Methodology) 71, 671-683.

Wang, D., Wu, T. and Zhao, Y. (2019). Penalized empirical likelihood for the sparse Cox regression model. Journal of Statistical Planning and Inference 201, 71-85.

Wood, A. T. A., Do, K. A. and Broom, N. M. (1996). Sequential linearization of empirical likelihood constraints with application to $U$-statistics. Journal of Computational and Graphical Statistics 5, 365385.

Zou, H. (2006). The adaptive LASSO and its oracle properties. Journal of the American Statistical Association 101, 1418-1429.

School of Mathematics and Statistics, Center for Data Science, Lanzhou University, Lanzhou 730000, China.

E-mail: lizhp@lzu.edu.cn

Zhejiang Institute of Research and Innovation, The University of Hong Kong, Hangzhou, China

Department of Statistics and Actuarial Science, The University of Hong Kong, Hong Kong, China.

E-mail: xujf@hku.hk

Department of Statistics and Actuarial Science, The University of Hong Kong, Hong Kong, China.

E-mail: zhaona@connect.hku.hk

Department of Statistics and Applied Probability, National University of Singapore, Singapore 
E-mail: stazw@nus.edu.sg 